\title{
ON A CONJECTURE OF M. S. ROBERTSON
}

\author{
ABDALLAH LYZZAIK
}

\begin{abstract}
We prove that two classes of univalent functions are equal. This settles a conjecture of $M$. S. Robertson in the affirmative.
\end{abstract}

1. Introduction. Recently, M. S. Robertson [3] introduced two classes of univalent functions, $\mathcal{G}$ and $\mathcal{G}^{*}$, and conjectured that they are equal. In this short note, we prove this conjecture.

First, let us define the classes $\mathcal{G}$ and $\mathcal{G}^{*}$.

Definition 1. Let $\mathcal{G}$ be the class of all functions $f$, regular and nonvanishing in $\mathbf{B}=\{z:|z|<1\}$, with $f(0)=1$, such that

$$
\operatorname{Re}\left\{2 z \frac{f^{\prime}(z)}{f(z)}+\frac{1+z}{1-z}\right\}>0 \text { for } z \in \mathbf{B} \text {. }
$$

Note that $1 \in \mathcal{G}$.

Let $D$ be a domain, and let $a$ belong to the closure of $D$. We say that $D$ is starlike with respect to $a$ if for each $z \in D$, every point $t z+(1-t) a$, with $0<t \leq 1$, belongs to $D$.

Definition 2. Let $\mathcal{G}^{*}$ be the class of functions $f$, regular and univalent in $\mathbf{B}$, with $f(0)=1$ and $\lim _{r \rightarrow 1^{-}} f(r)=0$, such that $f(\mathbf{B})$ is starlike with respect to the origin, and $\operatorname{Re}\left(e^{i a} f\right)>0$ for some real number $a$. Also, let $1 \in \mathcal{G}^{*}$.

For the sake of clarity, we remind the reader of some familiar definitions which are needed.

Definition 3. Let $S^{*}$ be the class of all functions $f$, regular in $\mathbf{B}$, with $f(0)=0$, such that

$$
\operatorname{Re} z \frac{f^{\prime}(z)}{f(z)}>0 \text { for } z \in \mathbf{B} \text {. }
$$

Note that there is no restriction on $f^{\prime}(0)$ in this definition.

It is known that each $f \in S^{*}$ is univalent and maps $\mathbf{B}$ onto a domain starlike with respect to the origin.

Definition 4. Let $S_{g}$ be the class of all functions $f$ which satisfy one of the following conditions:

(a) $f$ is regular and univalent in $\mathbf{B}$, and maps $\mathbf{B}$ onto a domain which contains the origin and is starlike with respect to the origin.

(b) $f$ is of the form

$$
f(z)=h(z) \frac{(z-\varsigma)(1-\bar{\zeta} z)}{z}, \quad|\zeta|<1,
$$

where $h \in S^{*}$.

Received by the editors March 3, 1983 and, in revised form, July 8, 1983.

1980 Mathematics Subject Classification. Primary 30C45.

Key words and phrases. Univalent functions, starlike functions. 
The equivalence of conditions (a) and (b) was shown by J. Hummel [1]. This will be our main tool for the proof.

\section{Proof of the conjecture.}

Theorem. $\mathcal{G}^{*}=\mathcal{G}$.

Before we give the proof, we remark that the set-inclusion $\mathcal{G} \subset \mathcal{G}^{*}$ was established by M. Robertson [3]. A short proof of this fact will be given as a part of the proof of the theorem.

Proof of Theorem. (a) $\mathcal{G}^{*} \subset \mathcal{G}$. Suppose that $f \in \mathcal{G}^{*}$ is not identical to 1 . It follows directly from Definition 2 that $f^{2}$ is univalent, $\lim _{r \rightarrow 1}-f(r)=0$, and $f$ maps $\mathbf{B}$ onto a domain starlike with respect to the origin. Let $D_{n}$ be the domain obtained from the union of the range of $f^{2}$ and the open disc centered at the origin and of radius $1 / n$. Evidently, each $D_{n}$ is simply connected. Let $f_{n}$ be a conformal map from $\mathbf{B}$ onto $D_{n}$ that satisfies $f_{n}(0)=1$ and $\arg f_{n}^{\prime}(0)=\arg \left(f^{2}\right)^{\prime}(0)$. By the Carathéodory Kernel Theorem [2, p. 29], $f_{n} \rightarrow f^{2}$ uniformly on compact subsets of B. From Definition 4, each $f_{n} \in S_{g}$. Hence for all $n$ we can write

$$
f_{n}(z)=h_{n}(z) \frac{\left(z-z_{n}\right)\left(1-\bar{z}_{n} z\right)}{z}, \quad\left|z_{n}\right|<1,
$$

where $h_{n} \in S^{*}$. It can be verified that

$$
f_{n}^{\prime}(0)=\frac{1}{2} \frac{h_{n}^{\prime \prime}(0)}{h_{n}^{\prime}(0)}+\left(1+\left|z_{n}\right|^{2}\right) h_{n}^{\prime}(0) .
$$

Since $f_{n}^{\prime}(0) \rightarrow\left(f^{2}\right)^{\prime}(0) \neq 0$ and $h \in S^{*}$ gives $\left|h_{n}^{\prime \prime}(0) / h_{n}^{\prime}(0)\right| \leq 4, h_{n}^{\prime}(0)$ is uniformly bounded for all $n$. Hence, there exists a sequence of positive integers $\left(n_{k}\right)$ so that $\left(h_{n_{k}}\right)$ converges uniformly on compact subsets to either $h \in S^{*}$ or zero. The latter case is impossible, otherwise $z f_{n} \rightarrow 0$ uniformly on compact subsets of $\mathbf{B}$ and $f$ will be identical to zero. Suppose that $z_{n_{k}} \rightarrow \zeta$, with $|\zeta| \leq 1$ (otherwise we choose a subsequence of $\left(z_{n_{k}}\right)$ that does so). Then we can write

$$
f^{2}(z)=h(z) \frac{(z-\varsigma)(1-\bar{\zeta} z)}{z}, \quad|\zeta| \leq 1
$$

Since $f$ does not admit zero in $\mathbf{B},|\zeta|=1$. Furthermore, since $\lim _{r \rightarrow 1}-f(r)=0$ and $h$ is bounded away from zero for values of $z$ close to $\partial \mathbf{B}, \varsigma=1$. Therefore,

which yields

$$
f^{2}(z)=-h(z) \frac{(1-z)^{2}}{z}
$$

$$
\operatorname{Re}\left\{2 z \frac{f^{\prime}(z)}{f(z)}+\frac{1+z}{1-z}\right\}=\operatorname{Re}\left\{z \frac{h^{\prime}(z)}{h(z)}\right\}>0
$$

and $f \in \mathcal{G}$.

(b) $\mathcal{G} \subset \mathcal{G}^{*}$. Let $f \in \mathcal{G}$, with $f$ not identical to 1 , and let $h(z)=f^{2}(z) z /(1-z)^{2}$. Then by simple calculation we have

So we have

$$
\operatorname{Re}\left\{z \frac{h^{\prime}(z)}{h(z)}\right\}=\operatorname{Re}\left\{2 z \frac{f^{\prime}(z)}{f(z)}+\frac{1+z}{1-z}\right\}>0 .
$$

$$
f^{2}(z)=h(z) \frac{(1-z)^{2}}{z},
$$

where $h \in S^{*}$, with $h^{\prime}(0)=1$. 
For every positive integer $n$, let $r_{n}=1-1 / n$, and let

$$
g_{n}(z)=-\frac{h(z)}{r_{n}} \frac{\left(z-r_{n}\right)\left(1-r_{n} z\right)}{z} .
$$

From Definition 4, each $g_{n} \in S_{g}$. Note that each $g_{n}(0)=1$, and

$$
g_{n}^{\prime}(0)=\frac{h^{\prime \prime}(0)}{2}-\frac{r_{n}^{2}+1}{r_{n}} \rightarrow \frac{h^{\prime \prime}(0)}{2}-2=\left(f^{2}\right)^{\prime}(0) \neq 0, \infty,
$$

since $h(z) \neq z /(1-z)^{2}$; otherwise $f$ is identically 1 . Also, note that $g_{n} \rightarrow f^{2}$ uniformly on compact subsets of B. By Hurwitz's Theorem, this implies the univalence of $f^{2}$ in $\mathbf{B}$. Let $\Delta=f^{2}(\mathbf{B})$, and let $\Delta_{n}=g_{n}(\mathbf{B})$. Then by the Carathéodory Kernel Theorem [2, p. 29], $\Delta_{n} \rightarrow \Delta$ as $n \rightarrow \infty$. Now we show that $\Delta$ is a domain starlike with respect to the origin. Let $w \in \Delta$. From the definition of the kernel [2, p. 28], there exists a domain $U$ and a positive integer $N$ such that $1, \dot{w} \in U$ and $U$ is contained in $\Delta_{n}$ for all $n>N$. Let $H$ be the domain consisting of all open-closed segments starting from the origin and ending in $U$. Since each $g_{n} \in S_{g}$, each $\Delta_{n}$ is starlike with respect to the origin. This implies that $H$ is contained in $\Delta_{n}$ for all $n>N$. Hence $H$ is contained in $\Delta$, and consequently $\Delta$ is starlike with respect to the origin. Since $0 \notin \Delta$, it is not hard to show that there exists a radial slit from the origin to infinity which does not meet $\Delta$. Hence, the univalence of $f^{2}$, and the starlikeness of $\Delta$ about the origin lead to the univalence of $f$, and to the starlikeness of $f(\mathbf{B})$ about the origin; moreover, there exists a real number $a$ such that $\operatorname{Re}\left(e^{i a} f\right)>0$ in $\mathbf{B}$. Since the origin is an accessible boundary point of $f(\mathbf{B})$, there exists $\zeta$, with $|\zeta|=1$, so that $\lim _{r \rightarrow 1^{-}} f(r \zeta)=0$ (see [2, p. 277]). Since $h$ is bounded away from zero for values of $z$ near $\partial \mathbf{B},(1)$ implies that $\varsigma=1$. Therefore, $f \in \mathcal{G}^{*}$ and the proof is complete.

The author can give an alternative shorter proof to the theorem based on $\mathrm{D}$. Styer [4]. However, this proof is quite involved, and was avoided for the sake of clarity.

The author thanks Professor Y. Abu-Muhanna for bringing the conjecture to his attention. Also, he thanks the University of Petroleum and Minerals for its support.

\section{REFERENCES}

1. J. A. Hummel, Multivalent starlike functions, J. Analyse Math. 18 (1967), 133-160.

2. Chr. Pommerenke, Univalent functions, Vandenhoeck \& Ruprecht, Göttingen, 1952.

3. M. S. Robertson, Univalent functions starlike with respect to a boundary point, J. Math. Anal. Appl. 81 (1981), 327-345.

4. D. D. Styer, On weakly starlike multivalent functions, J. Analyse Math. 26 (1973), 217-233.

Department of Mathematical Sciences, University of Petroleum and Minerals, Dhahran, Saudi Arabia 